\title{
The Schur multiplication in tensor algebras
}

\author{
by \\ ANNA MARIA MANTERO (Genova) \\ and \\ ANDREW TONGE (Cambridge and Orléans)
}

\begin{abstract}
W_{\theta}$ investigate the continuity of the Schur multiplication on the matrix algebras $l^{p} \otimes_{\varepsilon} l^{q}$ and on the tensor algebras $l^{p} \otimes_{8} l^{q} \otimes_{8} l^{r}$.
\end{abstract}

0. Introduction and notation. The central part of this article is devoted to a study of the so-called Schur multiplication of matrices and tensors. One of our main tools will be a probabilistic estimate for the norms of tensors whose coefficients are random \pm 1 's. This estimate generalises work of Bennett, Goodman and Newman [2], Carl, Maurey and Puhl [5] and Varopoulos [23]. In the final section, we show that the $r$-absolutely summing and $r$-nuclear norms of most random matrices behave like those of the Littlewood matrices considered by Pietsch [17].

We shall work with many rather recent results on Banach algebras, and so, in order to make the paper reasonably intelligible, we give a brief survey of the definitions and theorems needed later.

For us, a normed algebra is a normed space $A$ equipped with a continuous (associative) multiplication. In other words, there is some positive constant $K$ such that

$$
\|x y\| \leqslant K\|x\|\|y\| \quad \forall x, y \in A
$$

We do not necessarily suppose that $R$ is equal to 1 . In the sequel, we shall abusively use the letter $K$ to denote a constant independent of the dimension of any normed space with which it is associated. The value of $K$ may change from line to line.

We shall be especially interested in algebras derived from two important classes of Banach algebras.

(I) A uniform algebra is a closed subalgebra of the usual Banach algebra $C(X)$ of continuous functions on some compact Hausdorff space $X$. It does not necessarily have an identitzon 
(II) An operator algebra is a Banach algebra multiplicatively homeomorphic with a closed subalgebra of $L(H)$, the usual Banach algebra of bounded linear operators on some complex Hilbert space $H$. We do not postulate that it is closed under involution.

A Banach algebra homeomorphic with a quotient algebra of a uniform algebra is called a Q-algebra. It is an interesting and non-trivial fact that $Q$-algebras are operator algebras [25] and the same method shows that quotient algebras of operator algebras are operator algebras. $Q$-algebras were studied extensively by Davie [7] and Varopoulos [22], [23], and a useful theorem is

DAVIE'S ORITERION [7]. A commutative Banach algebra is a Q-algebra if and only if there is some positive $K$ such that

$$
\left\|\sum_{1 \leqslant k_{1}, \ldots, k_{J} \leqslant N} a(\boldsymbol{k}) x_{k_{1}} \ldots x_{k_{J}}\right\| \leqslant K^{J}\|a\|_{l^{1}(N) \otimes \check{\otimes} \ldots \check{\otimes}^{1}(N)}
$$

for all finite sequences $x_{1}, \ldots, x_{N}$ in ball $(A)$ and for all tensors a. (Here, and. later, $\boldsymbol{k}=\left(k_{1}, \ldots, k_{J}\right)$.)

Here, denotes the (completed) injective tensor product, as defined in [10], for example. Later, $\otimes_{8}$ will be used to denote the non-completed injective tensor product. For $1 \leqslant p \leqslant \infty$, we write $l^{p}(N)$ for the space $C^{N}$ equipped with the norm

$$
\|z\|_{p}=\|(z(1), \ldots, z(N))\|_{\nu_{(N)}}=\left(\sum_{n=1}^{N}|z(n)|^{p}\right)^{1 / p},
$$

with the usual convention when $p$ is infinite. The space of all $p$-summable sequences is written $l^{p}$, and it will be convenient to use the notation $b^{p}$ (or $b^{p}(N)$ ) for the unit ball of $l^{p}$ (or $l^{p}(N)$ ). If $a$ is a tensor, we shall often use the abbreviation

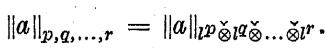

We shall always write $p^{\prime}=p /(p-1)$ for the index conjugate to $p$.

It follows from Grothendieck's inequality that all operator algebras satisfy Davie's criterion for homogeneous polynomials of degree two. Banach algebras with this property will be referred to as Hilbertian algebras.

The multiplication on a Banach algebra $A$ may be considered as a linear mapping $M: A \otimes A \rightarrow A$, and inequality (1) amounts to saying that $M$ is continuous when $A \otimes A$ is given the projective norm $\pi$ [10]. - It is interesting to consider more restrictive situations.

DEFInIIION. The Banach algebra $A$ is said to be an injective algebra if $M$ is continuous from $A \otimes_{8} A$ to $A$.

Varopoulos [22] proved that commutative injective algebras are $Q$-algebras. This result was extended by Charpentier [6], but, to describe his theorem, it is simpler to change our point of view. If $\varphi$ is in $A^{\prime}$, the dual of $A$, then we may define a linear mapping $\tilde{\varphi}: A \rightarrow A^{\prime}$ by

$$
\langle\tilde{\varphi}(x), y\rangle=\langle\varphi, x y\rangle \quad \forall x, y \in A .
$$

DEFINITION. The Banach algebra $A$ is said to be a p-summing algebra if there is a positive constant $K$ such that, for every $\varphi$ in $A^{\prime}$, the mapping $\tilde{\varphi}$ is $p$-summing and satisfies $\pi_{p}(\tilde{\varphi}) \leqslant K\|\varphi\|$.

We refer to [14] for the definition and basic properties of $p$-summing operators. Using the concepts of [13], one could define $(p, q, r)$-summing algebras in a similar way.

Charpentier's result is that a commutative 1-summing algebra is a $Q$-algebra. A related theorem states that a 2 -summing algebra is always an operator algebra [21].

Many of the positive results in this paper will depend on some littleknown inequalities due to Hardy and Littlewood. The key to the proof of these inequalities was the following lemma, which is a simple consequence of the definition of $\|\cdot\|_{p, q}$ and Khintchin's inequality.

LEMMA 0 (see [11]). If $1 \leqslant p, q \leqslant \infty$, then

$$
\sup \left\{\left(\sum_{m}\left(\sum_{k}|a(k, m) x(k)|^{2}\right)^{q / 2}\right)^{1 / q}: x \in b^{p^{\prime}}\right\} \leqslant K\|a\|_{p, q}
$$

and

$$
\sup \left\{\left(\sum_{k}\left(\sum_{m}|a(k, m) y(m)|^{2}\right)^{p / 2}\right)^{1 / p}: y \in b^{q^{\prime}}\right\} \leqslant K\|a\|_{p, q} .
$$

HaRdY-LITTLEWOOD INEQUALITIES [11]. Let $1 / p+1 / q>1$, and fix a in $l^{p} \ddot{\otimes} l^{q}$. Define $1 / u=1 / p+1 / q-1$.

(i) If $1 \leqslant p, q \leqslant 2$, then

$$
\left(\sum_{k}\left(\sum_{m}|a(k, m)|^{2}\right)^{u / 2}\right)^{1 / u} \leqslant K\|a\|_{p, q}
$$

and

$$
\left(\sum_{m}\left(\sum_{k}|a(k, m)|^{2}\right)^{u / 2}\right)^{1 / u} \leqslant K\|a\|_{p, q}
$$

(ii) We have

$$
\left(\sum_{k, m}|a(k, m)|^{u}\right)^{1 / u} \leqslant K \cdot\|a\|_{p, q},
$$

and if $1 / p+1 / q \geqslant 3 / 2$, then

$$
\left(\sum_{k, m}|a(k, m)|^{v}\right)^{1 / v} \leqslant K\|a\|_{p, q}
$$

where $2 / v=1 / p+1 / q-1 / 2$.

Note that the index $v$ is at most 2 . 
1. A probabilistic estimate for the norms of random tensors. The main theorem of this section is a probabilistic estimate for $\|t\|_{p_{1}, \ldots, p,}$, where $t\left(k_{1}, \ldots, k_{J}\right)$ is a random tensor of \pm 1 's. This will enable us to prove negative results about the Schur multiplication in matrix and tensor algebras.

THEOREM 1.1. Let $\left\{t\left(k_{1}, \ldots, k_{J}\right): 1 \leqslant k_{1}, \ldots, k_{J} \leqslant N\right\}$ be an independent family of random variables such that

Define

$$
\operatorname{prob}\left\{t\left(k_{1}, \ldots, k_{J}\right)=1\right\}=\operatorname{prob}\left\{t\left(k_{1}, \ldots, k_{J}\right)=-1\right\}=\frac{1}{2} .
$$

$$
f(p)=\left\{\begin{array}{lll}
1 / p-1 / 2 & \text { for } & 1 \leqslant p \leqslant 2, \\
0 & \text { for } & 2 \leqslant p \leqslant \infty .
\end{array}\right.
$$

Then, for all $0<\delta<1$ and for all positive integers $N$, we have

$$
\operatorname{prob}\left\{\|t\|_{p_{1}, \ldots, p_{J}} \leqslant K N^{\alpha(\boldsymbol{p})}\right\} \geqslant \delta,
$$

where $K$ is a constant independent of $N$ and

$$
\alpha(\boldsymbol{p})= \begin{cases}\max \left\{1 / p_{1}, \ldots, 1 / p_{J}\right\} & \text { if } 2 \leqslant p_{1}, \ldots, p_{J} \leqslant \infty, \\ 1 / 2+\sum_{j=1}^{J} f\left(p_{j}\right) & \text { if not. }\end{cases}
$$

Remark. An inspection of the proof of Theorem 1.1 will show that an identical result holds for symmetric random tensors.

The technique we use was developed by Varopoulos [23], and resembles the proof of the Kahane-Salem-Zygmund Theorem [12]. Varopoulos considered the case $J=3, p_{1}=p_{2}=p_{3}=2$, and he obtained a slightly weaker result than Theorem 1.1. He used his theorem to show the von Neumann inequality fails for polynomials in several commuting contractions on a complex Hilbert space. Theorem 1.1 may be applied to prove rather more than this in a much simpler way. [15].

In the case $J=2$, similar estimates have also been proved, independently of Varopoulos, by Bennett, Goodman and Newman [2] and by Carl, Maurey and Puhl [5]. In [2], certain operator ideals are identified by showing that if $t=(t(m, n))$ is an $M \times N$ matrix whose entries are mean zero independent random variables satisfying $|t(m, n)| \leqslant 1$ for all $m$ and $n$, then

$$
\text { (*) } \quad \boldsymbol{E}\left\{\|t\|_{l^{p}(M) \otimes l^{2}(N)}\right\} \leqslant K \cdot \max \left\{M^{1 / p}, N^{1 / 2}\right\} \quad(2 \leqslant p \leqslant \infty) .
$$

Here, $\boldsymbol{E}$ denotes mathematical expectation. Our theorem would also work under the above hypotheses on the coefficients of the tensor. In [5], the authors considered matrices $t=(t(m, n))$ whose entries were inde- pendent random variables such that

$$
\operatorname{prob}\{t(m, n)=1\}=\operatorname{prob}\{t(m, n)=-1\}=\frac{1}{2},
$$

and they found a better proof of the estimate $(*)$ in this special case In [15], Prop. 5, we use the proof of Theorem 1.1 to show that

$$
\boldsymbol{E}\left\{\|t\|_{p_{1}, \ldots, p_{J}}\right\} \leqslant K N^{\alpha(\boldsymbol{p})},
$$

where $\alpha(\boldsymbol{p})$ is defined by formula (2).

Theorem 1.1 is, in a rather strong sense, best possible.

THEOREM 1.2. If $J$ is a positive integer, and if $\alpha(\boldsymbol{p})$ is defined by formula (2), then

$$
\|t\|_{p_{1}, \ldots, p_{J}} \geqslant K N^{\alpha(\boldsymbol{p})}
$$

for all tensors $t(\boldsymbol{k})= \pm 1\left(1 \leqslant k_{1}, \ldots, k_{J} \leqslant N\right)$. Here, $K>0$ is a constant independent of $N$.

Proof of Theorem 1.1. If $a_{j} \in b^{p_{j}^{\prime}}(1 \leqslant j \leqslant J)$, we define

$$
T\left(x_{1}, \ldots, x_{J}\right)=\sum_{\boldsymbol{k}} t(\boldsymbol{k}) x_{1}\left(k_{1}\right) \ldots x_{J}\left(k_{J}\right) \text {. }
$$

Let $\||t|\|_{p_{1}, \ldots, p_{J}}$ denote the supremum of $\left|T\left(x_{1}, \ldots, x_{J}\right)\right|$ taken over all elements $x_{j}$ of the real unit ball of $l^{p_{j}^{\prime}}(N)(1 \leqslant j \leqslant J)$. It is clear that

$$
\|t\|_{p_{1}, \ldots, p_{J}} \leqslant 2^{J}\||| t\|_{p_{1}, \ldots, p_{J}} .
$$

Observe that if $\varepsilon<1$, then for each $1 \leqslant j \leqslant J$, the real unit ball of $l^{p_{j}^{\prime}}(N)$ may be covered by $M \leqslant((2+\varepsilon) / \varepsilon)^{N}$ real balls of radius $\varepsilon$, whose centres $c_{m, j}(1 \leqslant m \leqslant M)$ also lie in the real unit ball of $l^{p_{j}^{\prime}}(N)$. If we fix $x_{j}$ in the real unit ball of $l^{p_{j}^{\prime}}(N)$, we can thus choose $c_{m_{j}, j}$ such that

$$
\left\|x_{j}-c_{m_{j}, j}\right\|<\varepsilon \quad(1 \leqslant j \leqslant J) .
$$

By using the appropriate generalisation of the identity

we find that

$$
x y-a b=(x-a)(y-b)+a(y-b)+(x-a) b,
$$

$$
\left|T\left(x_{1}, \ldots, x_{J}\right)-T\left(c_{m_{1}, 1}, \ldots, c_{m_{J}, J}\right)\right| \leqslant K \varepsilon|||t| \|_{p_{1}, \ldots, p_{J}},
$$

where $K>1$ depends only on $J$. Now we define

$$
|t|_{p_{1}, \ldots, p_{J}}=\sup \left\{\left|T\left(c_{m_{1}, 1}, \ldots, c_{m_{J}, J}\right)\right|\right\},
$$

where the supremum is taken over all possible choices of $c_{m_{j}, j}\left(1 \leqslant m_{j} \leqslant M\right.$, $1 \leqslant j \leqslant J)$. Setting $\varepsilon=1 / 2 K$, we find that
(3)
$\|\left.|t|\right|_{p_{1}, \ldots, p_{J}} \leqslant 2|t|_{p_{1}, \ldots, p_{J}}$ 
Our next objective is to show that if $x_{j}$ is in the real unit ball of $l^{n_{j}^{\prime}}(N)$ $(1 \leqslant j \leqslant J)$, then

$$
\operatorname{prob}\left\{\left|T\left(x_{1}, \ldots, x_{J}\right)\right| \geqslant z\right\} \leqslant 2 \exp \left(-z^{2} N^{1-2 \alpha(\boldsymbol{p})} / 2\right)
$$

provided that not all the $p_{j}$ are greater than 2 .

To see this, note that, on taking expectations, we have

$$
\begin{aligned}
\boldsymbol{E}\{\exp (\lambda T & \left.\left.\left(x_{1}, \ldots, x_{J}\right)\right)\right\}=\prod_{\gamma_{i}} \cosh \left(\lambda x_{1}\left(k_{1}\right) \ldots x_{J}\left(k_{J}\right)\right) \\
& \leqslant \exp \left(\lambda^{2} \sum_{l_{l}}\left(x_{1}\left(k_{1}\right) \ldots x_{J}\left(k_{J}\right)\right)^{2} / 2\right)=\exp \left(\lambda^{2}\left\|x_{1}\right\|_{2}^{2} \ldots\left\|x_{J}\right\|_{2}^{2} / 2\right) \\
& \leqslant \exp \left(\lambda^{2} N^{2 a(p)-1} / 2\right),
\end{aligned}
$$

since

$$
\left\|x_{j}\right\|_{2} \leqslant N^{f\left(p_{j}\right)}\left\|x_{j}\right\|_{p_{j}^{\prime}} \leqslant N^{f\left(p_{j}\right)}
$$

Chebysher's inequality now yields that

$$
\operatorname{prob}\left\{\left|T\left(x_{1}, \ldots, x_{J}\right)\right| \geqslant z\right\} \leqslant 2 \exp \left(-\lambda z+\frac{1}{2} \lambda^{2} N^{2 \alpha(\boldsymbol{p})-1}\right)
$$

for all positive $\lambda$. Setting $\lambda=z N^{1-2 a(p)}$, we have proved (4).

\section{Consequently}

$$
\begin{aligned}
\operatorname{prob}\left\{\|t\|_{p_{1}, \ldots, p_{J}} \geqslant 2^{J+1} z\right\} & \leqslant \operatorname{prob}\left\{\||| t||_{p_{1}, \ldots, p_{J}} \geqslant 2 z\right\} \\
& \leqslant \operatorname{prob}\left\{|t|_{p_{1}, \ldots, p_{J}} \geqslant z\right\} \quad(\text { by }(3)) \\
& \leqslant(1+4 K)^{N J} \cdot 2 \exp \left(-z^{2} N^{1-2 \alpha(\boldsymbol{p})} / 2\right) .
\end{aligned}
$$

(To obtain the last inequality, replace the probability of the supremum by the sum of the probabilities, and use (4).) On setting $z^{2}=N^{2 a(p)} .4 J$. . $\log ((1+4 K) /(1-\delta))$, we find the conclusion of the theorem, except in the case where $2<p_{1}, \ldots, p_{J} \leqslant \infty$.

To consider this final case, it is no loss of generality to suppose that $p_{1}=\min \left(p_{1}, \ldots, p_{J}\right)$. Noting that $\|t\|_{\infty, \ldots, \infty}=1$, we may use the complex interpolation method to deduce that

Hence

$$
\begin{aligned}
\|t\|_{2, \ldots, 2} \leqslant K N^{1 / 2} & \Rightarrow\|t\|_{p_{1}, \ldots, p_{1}} \leqslant K^{2 / p_{1}} N^{1 / p_{1}} \\
& \Rightarrow\|t\|_{p_{1}, \ldots, p_{J}} \leqslant K^{2 / p_{1}} N^{1 / p_{1}}
\end{aligned}
$$

$$
\operatorname{prob}\left\{\|t\|_{p_{1}, \ldots, p_{J}} \leqslant K^{2 / p_{1}} N^{1 / p_{1}}\right\} \geqslant \operatorname{prob}\left\{\|t\|_{2, \ldots, 2} \leqslant K N^{1 / 2}\right\},
$$

and the conclusion follows at once.
Proof of Theorem 1.2. This is obvious if $J=1$.

(a) $J=2$.

$$
\begin{aligned}
\|t\|_{p_{1}, p_{2}} & =\sup \left\{\left|\sum_{k_{1}, k_{2}} t\left(k_{1}, k_{2}\right) x\left(k_{1}\right) y\left(k_{2}\right)\right|: x \in b^{p_{1}^{\prime}}, y \in b^{p_{2}^{\prime}}\right\} \\
& \geqslant \sup \left\{\left|\sum_{k_{1}} t\left(k_{1}, k_{2}\right) x\left(k_{1}\right)\right|: x \in b^{p_{1}^{\prime}}, 1 \leqslant k_{2} \leqslant N\right\} \\
& \geqslant \sup _{k_{2}}\left(\sum_{k_{1}}\left|t\left(k_{1}, k_{2}\right)\right|^{p_{1}}\right)^{1 / p_{1}}=N^{1 / p_{1}}
\end{aligned}
$$

Similarly, $\|t\|_{p_{1}, p_{2}} \geqslant N^{1 / p_{2}}$, and so the theorem is proved, except in the case where $1 \leqslant p_{1}, p_{2}<2$. However, under these conditions, the result is a simple consequence of Lemma 0 . Note first that, since $t\left(k_{1}, k_{2}\right)^{2}=1$ for all $k_{1}$ and $k_{2}$, we have

$$
\left\|t^{2}\right\|_{p_{1}, p_{2}}=N^{1 / p_{1}+1 / p_{2}}
$$

Then, applying the definition of the norm $\|\cdot\|_{p_{1}, p_{2}}$ and the duality between $l^{p}(N)$ and $l^{p^{\prime}}(N)$, it follows that

$$
\begin{aligned}
N^{1 / p_{1}+1 / p_{2}} & =\sup \left\{\left|\sum_{k_{1}, k_{2}} t\left(k_{1}, k_{2}\right)^{2} x\left(k_{1}\right) y\left(k_{k_{2}}\right)\right|: x \in b^{p_{1}^{\prime}}, y \in b^{p_{2}^{\prime}}\right\} \\
& =\sup _{x}\left[\sum_{k_{2}}\left|\sum_{k_{1}} t\left(k_{1}, k_{2}\right)^{2} x\left(k_{1}\right)\right|^{p_{2}}\right]^{1 / p_{2}} \\
& \leqslant \sup _{x}\left[\sum_{k_{2}}\left(\left.\sum_{k_{1}} t\left(k_{1}, k_{2}\right)^{2}\right|^{p_{2} / 2} \cdot\left(\sum_{k_{1}}\left|t\left(k_{1}, k_{2}\right) x\left(k_{1}\right)\right|^{2}\right)^{p_{2} / 2}\right]^{1 / p_{2}}\right. \\
& =N^{1 / 2} \cdot \sup _{x}\left[\sum_{k_{2}}\left(\sum_{k_{1}}\left|t\left(k_{1}, k_{2}\right) x\left(k_{1}\right)\right|^{2}\right)^{p_{2} / 2}\right]^{1 / p_{2}} \\
& \leqslant K N^{1 / 2}\|t\|_{p_{1}, p_{2}} \quad(\text { by Lemma } 0) .
\end{aligned}
$$

We deduce at once that $\|t\|_{p_{1}, p_{2}} \geqslant K N^{1 / p_{1}+1 / p_{2}-1 / 2}$.

(b) $J>2$. Here, we work inductively. We suppose that the result is known for tensors of order less than $J$ and we prove the required inequality for a tensor $\left(t\left(k_{1}, \ldots, k_{J}\right)\right)$ of order $J$.

(i) We show that if at least one of the indices is $\geqslant 2$, then the estimate is an immediate consequence of the inductive hypothesis. Suppose without loss of generality that $p_{1} \geqslant 2$ is the largest index. Define tensors $T_{k}(1 \leqslant k \leqslant N)$ by

Then

$$
T_{k}\left(k_{2}, \ldots, k_{J}\right)=t\left(k, k_{2}, \ldots, k_{J}\right)
$$

$\|t\|_{p_{1}, \ldots, p_{J}} \geqslant\|t\|_{\infty, p_{2}, \ldots, p_{J}}=\sup _{k}\left\|T_{k}\right\|_{p_{2}, \ldots, p_{J}} \geqslant K N^{\alpha\left(p_{2}, \ldots, p_{J}\right)}=K N^{a\left(p_{1}, \ldots, p_{J}\right)}$ by hypothesis. 
(ii) The remaining case is where $1 \leqslant p_{1}, \ldots, p_{J}<2$. To deal with this, it is convenient to establish a generalisation of Lemma 0 . Notice that, if $x_{j} \in b^{p_{j}^{\prime}}(1 \leqslant j \leqslant J)$, then

$$
\sum_{k_{1}}\left|\sum_{k_{2}, \ldots, k_{J}} t\left(k_{1}, \ldots, k_{J}\right) x_{2}\left(k_{2}\right) \ldots x_{J}\left(k_{J}\right)\right|^{p_{1}} \leqslant\|t\|_{p_{1}, \ldots, p_{J}}^{p_{1}}
$$

Replacing $x_{j}\left(k_{j}\right)$ by $x_{j}\left(k_{j}\right) r_{k_{j}}\left(s_{j}\right)$, where $r_{k}$ denotes the $k$ th Rademacher function, and integrating with respect to the $s_{j}$ 's, we obtain

$\sum_{k_{1}} \int \ldots \int\left|\sum_{k_{2}, \ldots, k_{J}} t(\boldsymbol{R}) x_{2}\left(k_{2}\right) \ldots x_{J}\left(k_{J}\right) r_{k_{2}}\left(s_{2}\right) \ldots r_{k_{J}}\left(s_{J}\right)\right|^{p_{1}} d s_{2} \ldots d s_{J} \leqslant\|t\|_{p_{1}, \ldots, p_{J}}^{p_{1}}$

Now the multidimensional Khintchin inequality states that the $L^{p}$ norm of a Rademacher sum is equivalent to the $L^{2}$ norm. Using the orthonormality of the Rademacher functions, we deduce that

$$
\sum_{k_{1}}\left[\sum_{k_{2}, \ldots, k_{J}}\left|t(\boldsymbol{k}) x_{2}\left(k_{2}\right) \ldots x_{J}\left(k_{J}\right)\right|^{2}\right]^{p_{1} / 2} \leqslant K\|t\|_{p_{1}, \ldots, p_{J}}^{p_{1}}
$$

From this point, we proceed just as in the case $J=2$, and we find that

$$
N^{1 / p_{1}+\ldots+1 / p_{J}}=\left\|t^{2}\right\|_{p_{1}, \ldots, p_{J}} \leqslant K N^{(J-1) / 2}\|t\|_{p_{1}, \ldots, p_{J}},
$$

which is simply a restatement of the result we wish to prove.

2. The Schur multiplication in matrix and tensor algebras. It is well known that $l^{p}(1 \leqslant p \leqslant \infty)$ is a Banach algebra under the pointwise multiplication defined by

$$
(x(n)) \cdot(y(n))=(x(n) y(n)) \quad \forall x, y \in l^{p} .
$$

Varopoulos [22] proved the stronger result that $l^{p} .(1 \leqslant p \leqslant \infty)$ is in fact a $Q$-algebra. The pointwise multiplication on $l^{p}$ and $l^{q}(1 \leqslant p, q \leqslant \infty)$ may be used to define the Schur multiplication on $l^{p} \otimes_{8} l^{q}$. This is generated by

$$
\left(x_{1} \otimes y_{1}\right) \cdot\left(x_{2} \otimes y_{2}\right)=x_{1} x_{2} \otimes y_{1} y_{2} \quad \forall x_{1}, x_{2} \in l^{p}, \forall y_{1}, y_{2} \in l^{\alpha} .
$$

In terms of matrices, this multiplication is given by

$$
(a(m, n)) \cdot(b(m, n))=(a(m, n) b(m, n)) \quad \forall a, b \in l^{p} \otimes_{\theta} l^{\alpha} .
$$

Schur [20] proved that $l^{2} \otimes_{\varepsilon} l^{2}$ is a normed algebra, and this implies, by continuity, that $l^{2} \ddot{\otimes} l^{2}$ is a Banach algebra. Varopoulos [ $\left.\overline{2} 3\right]$ went further to show that $l^{2} \ddot{\otimes} l^{2}$ is an operator algebra. He also asked whether $l^{2} \otimes l^{2}$ is a $Q$-algebra. Although we cannot answer this question, we are able to prove results on the structure of $l^{2} \check{\otimes} l^{2}$, and, more generally, of $l^{p} \check{\otimes} l^{q}$ $(1 \leqslant p, q \leqslant \infty)$. The starting point is
Proposition 2.1. $l^{p} \otimes_{\varepsilon} l^{q}$ is a normed algebra for all $1 \leqslant p, q \leqslant \infty$.

Proof. (a) $1 / p+1 / q \leqslant 1$. This is the elementary case, and we will show that the multiplication has norm $\leqslant 1$. Take $a$ and $b$ in the unit ball of $l^{p} \otimes_{\varepsilon} l^{q}$. Then

(5) $\quad \sup _{n}\left(\sum_{m} \cdot|a(m, n)|^{p}\right)^{1 / p} \leqslant 1 \quad$ and $\quad \sup _{m}\left(\sum_{n}|b(m, n)|^{q}\right)^{1 / q} \leqslant 1$.

We have

$$
\begin{aligned}
\|a b\|_{p, q} & =\sup _{x}\left\{\left(\sum_{m}\left|\sum_{n} a(m, n) b(m, n) y(n)\right|^{p}\right)^{1 / p}: y \in b^{q^{\prime}}\right\} \\
& \leqslant \sup _{v}\left[\sum_{m}\left(\sum_{n}|a(m, n) y(n)|^{q^{\prime}}\right)^{p / q^{\prime}} \cdot\left(\sum_{n}|b(m, n)|^{q}\right)^{p / q}\right]^{1 / p} \\
& \leqslant \sup _{v}\left[\sum_{n}\left(\sum_{m}|a(m, n) y(n)|^{p}\right)^{q^{\prime} / p}\right]^{1 / q^{\prime}}
\end{aligned}
$$

(using (5) and Minkowski's inequality (since $q^{\prime} \leqslant p$ ))

$$
=\sup _{y}\left[\sum_{n}|y(n)|^{q^{\prime}}\left(\sum_{m}|a(m, n)|^{p}\right)^{q^{\prime} / p}\right]^{1 / q^{\prime}} \leqslant 1 \quad \text { (using (5) again). }
$$

(b) $1 / p+1 / q>1$. Here, we are not able to prove that the multiplication is a contraction. We may, without loss of generality, suppose that $q \leqslant 2$. Then, if $a$ and $b$ are in ball $\left(l^{p} \otimes_{\sigma} l^{q}\right)$, we have

$$
\begin{aligned}
\|a b\|_{p, q} & =\sup \left\{\left(\sum_{m}\left|\sum_{n} a(m, n) b(m, n) y(n)\right|^{p}\right)^{1 / p}: y \in b^{q^{\prime}}\right\} \\
& \leqslant \sup _{y}\left[\sum_{m}\left(\sum_{n}|a(m, n) y(n)|^{2}\right)^{p / 2} \cdot\left(\sum_{n}|b(m, n)|^{2}\right)^{p / 2}\right]^{1 / p} \\
& \left.\leqslant \sup _{y}\left[\sum_{m}\left(\sum_{n}|a(m, n) y(n)|^{2}\right)^{p / 2}\right]^{1 / p} \quad \text { (by (5), since } q \leqslant 2\right) \\
& \leqslant K \quad(\text { by Lemma 0). }
\end{aligned}
$$

Going on from here, we have the much more precise

THEOREM 2.2. $l^{p} \check{\otimes} l^{q}$ is a Hilbertian algebra for all $1 \leqslant p, q \leqslant \infty$.

Proof. Take $a_{1}, \ldots, a_{J}$ in the unit ball of $l^{p} \otimes l^{q}$. Then we must show that, for some positive $K$, we have

$$
\left\|\sum_{i, j} t(i, j) a_{i} a_{j}\right\|_{p, q} \leqslant K\|t\|_{1,1}
$$

for all matrices $(t(i, j): 1 \leqslant i, j \leqslant J)$. Fix $x \in b^{p^{\prime}}$ and $y \in b^{q}$, and define

$$
A_{j}(m, n)=a_{j}(m, n) x(m)^{1 / 2} y(n)^{1 / 2} \quad(1 \leqslant j \leqslant J) .
$$


(The precise meaning of $x(m)^{1 / 2}$ will be unimportant.) Then

$$
\begin{aligned}
\sum_{m, n}\left|A_{j}(m, n)\right|^{2} & =\sum_{m, n} a_{j}(m, n) \overline{a_{j}(m, n)}|x(m) y(n)| \\
& \leqslant K\left\|a_{j}\right\|_{p, q}\left\|\overline{a_{j}}\right\|_{p, q} \quad \text { (by Proposition 2.1) } \\
& \leqslant K .
\end{aligned}
$$

Consequently, $A_{j}=\left(A_{j}(m, n)\right)$ may be considered as an element of $l^{2}\left(\boldsymbol{Z}^{+} \times \boldsymbol{Z}^{+}\right)$of norm bounded by $K^{1 / 2}(1 \leqslant j \leqslant J)$. It follows from Grothendieck's inequality that

$$
\left|\sum_{i, j, m, n} t(i, j) a_{i}(m, n) a_{j}(m, n) x(m) y(n)\right| \leqslant K K_{G}\|t\|_{1,1},
$$

and, since this is true for all $x \in b^{p^{\prime}}$ and $y \in b^{q^{\prime}}$, the proof is complete.

Using Blei's generalisation [3] of Grothendieck's inequality, and the Hardy-Littlewood inequalities, the same process allows us to determine regions where Davie's criterion is satisfied for homogeneous polynomials of degree greater than two. As an example, we state without proof

PROPOSTrion 2.3. $l^{p} \otimes l^{\alpha}$ satisfies Davie's criterion for polynomials of degree 3 if $1 \leqslant p, q \leqslant 2$ and $2 / p+1 / q \geqslant 2$ or $1 / p+2 / q \geqslant 2$.

This corresponds to the shaded region in the diagram below.

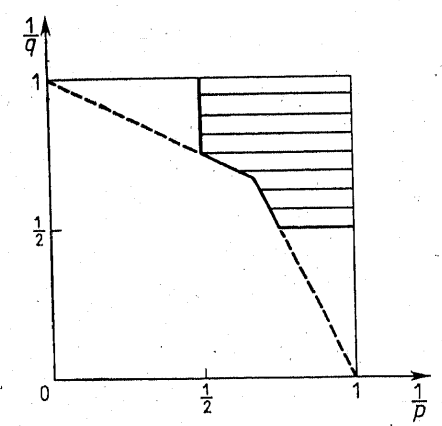

One way of showing that a commutative Banach algebra is a $Q$-algebra is to prove that it is a 1-summing algebra.

THEOREM 2.4. $l^{p} \ddot{\otimes} l^{\alpha}$ is a 1-summing algebra if and only if

(a) $1 / p+1 / q \geqslant 3 / 2$

or

(b) $1 \leqslant p \leqslant 2, q=\infty$, or $p=\infty, 1 \leqslant q \leqslant 2$ or $p=q=\infty$.

$l^{p} \otimes l^{q}$ is thus a 1 -summing algebra in the heavily shaded regions of the following diagram, and only in those regions.

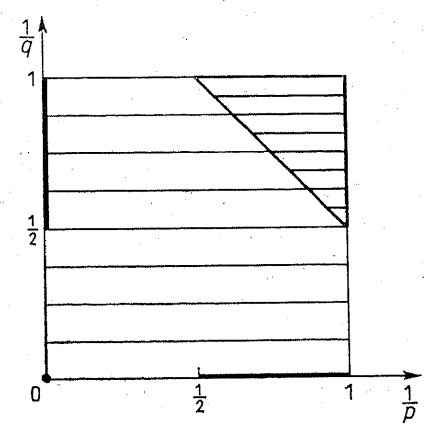

We shall use several lemmas to prove this theorem.

LEMMA 2.5. Let $A$ be an injective algebra, let $R$ be a commutative Banach algebra, and suppose that $A \otimes 2$ has the induced multiplication.

(i) If $R$ is a 1-summing algebra, then so is $A \ddot{\otimes} R$.

(ii) If $R$ is a $Q$-algebra, then so is $A \ddot{\otimes} R$.

The extremal case (b) of Theorem 2.4 follows at once from this lemma if we note that $l^{1}$ and $l^{\infty}$ are injective algebras [22] and that $l^{p}(1 \leqslant p \leqslant 2)$ is a 1-summing algebra [6]. We remark in [16] that $l^{p}$ is not injective for $p \neq 1$ or $\infty$.

Proof of Lemma 2.5. Varopoulos [24] has shown that $A \bowtie r R$ is indeed a Banach algebra. We only propose to prove part (i). The interested reader will have no difficulty in completing part (ii). It is enough to find some positive $K$ such that, if $x_{1}, \ldots, x_{N}$ in $A \otimes_{\varepsilon} R$ satisfy

$$
\sup \left\{\sum_{n}\left|\left\langle x_{n}, F\right\rangle\right|: F \in \operatorname{ball}\left((A \ddot{\otimes} R)^{\prime}\right)\right\} \leqslant 1
$$

and if $y_{1}, \ldots, y_{N}$ are in $\operatorname{ball}\left(A \otimes_{\varepsilon} R\right)$, then $\left\|\sum_{n} x_{n} y_{n}\right\| \leqslant K$.

Suppose that $x_{n}=\sum_{i} a_{n i} \otimes r_{n i}$ and $y_{n}=\sum_{j} b_{n j} \otimes s_{n j}$. Then

$$
\begin{aligned}
\left\|\sum_{n} x_{n} y_{n}\right\| & =\sup \left\{\left\|\sum_{i, j, n}\left\langle a_{n i} b_{n j}, u\right\rangle r_{n i} s_{n j}\right\|: u \in \operatorname{ball}\left(A^{\prime}\right)\right\} \\
& \leqslant K \sup _{u}\left\|\int_{n} \sum_{i}\left(\sum_{i}\left\langle a_{n i}, v\right\rangle r_{n i}\right) \cdot\left(\sum_{j}\left\langle b_{n j}, w\right\rangle s_{n j}\right) d \lambda_{u}(v, w)\right\|
\end{aligned}
$$

(where $\lambda_{u}$ is a probability measure on $\operatorname{ball}\left(A^{\prime}\right) \times \operatorname{ball}\left(A^{\prime}\right)[22]$ )

$$
\leqslant K \sup \left\{\sum_{n}\left|\sum_{i}\left\langle a_{n i}, v\right\rangle\left\langle r_{n i}, r^{\prime}\right\rangle\right|:\|v\|_{A^{\prime}} \leqslant 1,\left\|r^{\prime}\right\|_{R^{\prime}} \leqslant 1\right\} .
$$

(since $R$ is a 1 -summing algebra and $\left\|y_{n}\right\| \leqslant 1(1 \leqslant n \leqslant N)$ )

$$
\leqslant K
$$

(by the hypothesis on the $x_{n}$ 's). 
To decide when $l^{p} \check{\otimes} l^{q}$ is not a 1 -summing algebra, we shall need to use the probabilistic estimates of Section 1, and

LEMMA 2.6. $l^{p}(2<p<\infty)$ is not a 1-summing algebra.

in conjunction with

LEMna 2.7. Let $A$ and $B$ be Banach algebras with a non-trivial multiplication. Suppose that $A \check{\otimes} B$ is an r-summing algebra under the induced multiplication. Then $A$ and $B$ must both be r-summing algebras.

Lemma 2.6 incidentally answers a question in [6]. The proof, as we pointed out in [16], is a simple reinterpretation of Garling's result [8] that, for $2<p<\infty$, the diagonal mapping

$$
l^{p} \rightarrow l^{p^{\prime}} ; \quad(x(n)) \mapsto(d(n) x(n))
$$

is 1-summing if and only if

$$
\sum_{n}|d(n)|^{p^{\prime}}(1+|\log (|d(n)|)|)<\infty .
$$

Proof of Lemma 2.7. It will be enough to show that $A$ is an $r$-summing algebra. Accordingly, fix $b$ and $\beta$ of norm 1 in $B$ such that $b \beta \neq 0$, and choose $x_{n}=f_{n} \otimes b \in A \check{\otimes} B$ and $y_{n}=g_{n} \otimes \beta \in \operatorname{ball}(A \check{\otimes} B)$. There is some $b^{\prime} \in$ ball $\left(B^{\prime}\right)$ such that $\left\langle b \beta, b^{\prime}\right\rangle \neq 0$. Take $a^{\prime} \in \operatorname{ball}\left(A^{\prime}\right)$ and write $F=a^{\prime} \otimes b^{\prime}$. Since

$$
\begin{aligned}
\left(\sum_{n}\left\|\tilde{W^{\prime}}\left(x_{n}\right)\right\|^{r}\right)^{1 / r} & \leqslant K \sup \left\{\left(\sum_{n}\left|\left\langle x_{n}, G\right\rangle\right|^{r}\right)^{1 / r}:\|G\|_{(A \dot{\otimes} B)^{\prime}} \leqslant 1\right\} \\
& \leqslant K \sup \left\{\left(\sum_{n}\left|\left\langle f_{n}, \alpha^{\prime}\right\rangle\left\langle b, \beta^{\prime}\right\rangle\right|^{r}\right)^{1 / r}:\left\|\alpha^{\prime}\right\| \leqslant 1,\left\|\beta^{\prime}\right\| \leqslant 1\right\} \\
& \leqslant K \sup \left\{\left(\sum_{n}\left|\left\langle f_{n}, \alpha^{\prime}\right\rangle\right|^{r}\right)^{1 / r}:\left\|\alpha^{\prime}\right\| \leqslant 1\right\}
\end{aligned}
$$

we must have

$$
\left(\sum_{n}\left|\left\langle x_{n} y_{n}, H^{\prime}\right\rangle\right|^{r}\right)^{1 / r} \leqslant K \sup \left\{\left(\sum_{n}\left|\left\langle f_{n}, \alpha^{\prime}\right\rangle\right|^{r}\right)^{1 / r}: \quad\left\|\alpha^{\prime}\right\| \leqslant 1\right\} .
$$

Consequently, as $\left\langle b \beta, b^{\prime}\right\rangle \neq 0$,

$$
\left(\sum_{n}\left|\left\langle f_{n} g_{n}, a^{\prime}\right\rangle\right|^{r}\right)^{1 / r} \leqslant \mathcal{K} \sup \left\{\left(\sum_{n}\left|\left\langle f_{n}, \alpha^{\prime}\right\rangle\right|^{r}\right)^{1 / r}: \quad\left\|\alpha^{\prime}\right\| \leqslant 1\right\} .
$$

This is true for all $f_{n}$ in $A$ and for all $g_{n}$ in ball $(A)$, so we conclude that $A$ is an $r$-summing algebra.

We are now in a position to give the

Proof of Theorem 2.4.

(i) Positive results. We have already disposed of case (b), so let us take $1 / p+1 / q \geqslant 3 / 2$. We must show that, for some positive $K$,

(6) $\sup \left\{\sum_{j=1}^{J}\left|\sum_{m, n} a_{j}(m, n) b_{j}(m, n) x(m) y(n)\right|: x \in b^{p^{\prime}}, y \in b^{Q^{\prime}}\right\} \leqslant K$

for every finite sequence $a_{1}, \ldots, a_{J}$ in $l^{p} \check{\otimes} l^{q}$ satisfying

$$
\sup \left\{\sum_{j=1}^{J}\left|\sum_{m, n} a_{j}(m, n) x(m) y(n)\right|: x \in b^{p^{\prime}}, y \in b^{q^{\prime}}\right\} \leqslant 1
$$

and for every finite sequence $b_{1}, \ldots, b_{J}$ in $\operatorname{ball}\left(l^{p} \check{\otimes} l^{\alpha}\right)$.

If we replace $x(m)$ and $y(n)$ in (7) by $x(m) r_{m}(t)$ and $y(n) r_{n}\left(t^{\prime}\right)$, where $r_{m}(t)$ and $r_{n}\left(t^{\prime}\right)$ are Rademacher functions, then we may integrate inequality (7), and then use Khintchin's inequality to obtain

$$
\sup _{x, y} \sum_{j=1}^{J}\left(\sum_{m, n}\left|a_{j}(m, n) x(m) y(n)\right|^{2}\right)^{1 / 2} \leqslant K
$$

for some constant $K$. On the other hand, the hypothesis that $1 / p+1 / q \geqslant 3 / 2$ allows us to use the Hardy-Littlewood inequalities to deduce

$$
\left(\sum_{m, n}\left|b_{j}(m, n)\right|^{2}\right)^{1 / 2} \leqslant K \quad(1 \leqslant j \leqslant J) .
$$

It follows from (8) and (9) that

$$
\begin{aligned}
\sup _{x, y} \sum_{j} \mid & \sum_{m, n} a_{j}(m, n) b_{j}(m, n) x(m) y(n) \mid \\
& \leqslant \sup _{x, y} \sum_{j}\left(\sum_{m, n}\left|a_{j}(m, n) x(m) y(n)\right|^{2}\right)^{1 / 2} \cdot\left(\sum_{m, n}\left|b_{j}(m, n)\right|^{2}\right)^{1 / 2} \leqslant K .
\end{aligned}
$$

(ii) Negative results. If either $2<p<\infty$ or $2<q<\infty$, then Lemmas 2.6 and 2.7 yield that $l^{p} \ddot{\otimes} l^{q}$ is not a 1 -summing algebra. We therefore consider the case $1 \leqslant p, q \leqslant 2,1 / p+1 / q<3 / 2$. It follows from (6) that for $l^{p} \otimes l^{q}$ to be a 1 -summing algebra, there must be a positive $K$ (independent of $N$ ) for which

$$
\begin{aligned}
\|a b\|_{1, p, q} & \leqslant K\|a\|_{1, p, q} \sup \left\{\left\|b_{j}\right\|_{p, q}: 1 \leqslant j \leqslant N\right\} \\
& =K\|a\|_{1, p, q}\|b\|_{\infty, p, q}
\end{aligned}
$$

for all $N$ and for all tensors $a=\left(a_{j}(m, n): 1 \leqslant j, m, n \leqslant N\right)$ and $b$ $=\left(b_{j}(m, n): 1 \leqslant j, m, n \leqslant N\right)$. However, Theorem 1.1 allows us to choose $a_{j}(m, n)=b_{j}(m, n)= \pm 1$ such that

$$
\|a\|_{1, p, q} \leqslant K N^{1 / p+1 / q} \text { and }\|b\|_{\infty, p, q} \leqslant K N^{1 / p+1 / q-1 / 2} \text {. }
$$

Using the fact that $a_{j}(m, n) b_{j}(m, n)=1$, we find that

$$
\|a b\|_{1, p, q}=N^{1+1 / p+1 / q} \text {. }
$$


Consequently, (10) can only be satisfied if

$$
N^{1+1 / p+1 / \alpha} \leqslant K N^{2 / p+2 / q-1 / 2}
$$

for every positive integer $N$. Since this cannot be true for $1 / p+1 / q<3 / 2$, we have the required result.

As $l^{p}(1 \leqslant p \leqslant \infty)$ is a $Q$-algebra, Lemma 2.5 (ii) enables us to deduce the following

Corollary to Theorem 2.4. $l^{p} \otimes l^{\alpha}$ is a Qalgebra if $1 / p+1 / q \geqslant 3 / 2$, or if either $p$ or $q$ is 1 or $\infty$.

Since every 2-summing algebra is an operator algebra, we are prompted to try to decide when $l^{p} \dot{\otimes} l^{q}$ has this property.

Proposimion 2.8. (a) $l^{p} \ddot{\otimes} l^{q}$ is a 2-summing algebra if $1 / p+1 / q \geqslant 3 / 2$, or if either $p$ or $q$ is 1 or $\infty$.

(b) $l^{p} \check{\otimes} l^{q}$ is not a 2-summing algebra if $2 \leqslant p, q<\infty$, or if

or

$$
1 / p+1 / q<3 / 2 \quad(1<p \leqslant 2,1<q \leqslant 2)
$$

$$
1 / p-1 / q<1 / 2 \quad(1<p \leqslant 2,2 \leqslant q<\infty)
$$

$$
1 / q-1 / p<1 / 2 \quad(2 \leqslant p<\infty, 1<q \leqslant 2) .
$$

Diagrammatically, we have the situation below. The white regions are areas of uncertainty.

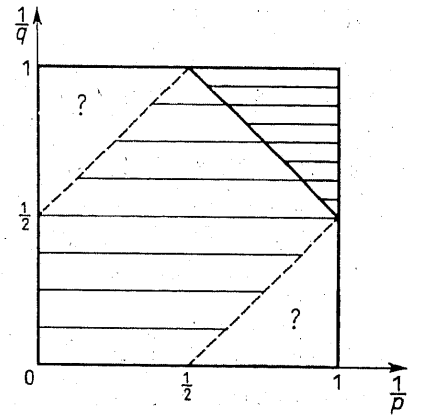

Proof. (a) The case $1 / p+1 / q \geqslant 3 / 2$ is covered by Theorem 2.4. The extremal cases are treated by observing that $l^{p}(1 \leqslant p \leqslant \infty)$ is a 2 -summing algebra, and by proving the necessary analogue of Lemma 2.5.

(b) As in the proof of Theorem 2.4, $l^{p} \otimes l^{q}$ can only be a 2 -summing algebra if there is some positive $K$ (independent of $N$ ) for which

$$
\|a b\|_{2, p, q} \leqslant K\|a\|_{2, p, q}\|b\|_{\infty, p, q}
$$

for all tensors $a=(a(j, m, n): 1 \leqslant j, m, n \leqslant N)$ and $b=(b(j, m, n)$ : $1 \leqslant j, m, n \leqslant N)$ and for all $N$. However, by Theorem 1.1, we can take $a(j, m, n)=b(j, m, n)= \pm 1$ such that

$$
\|a\|_{2, p, q} \leqslant K \begin{cases}N^{1 / p+1 / q-1 / 2} & (1<p \leqslant 2,1<q \leqslant 2) \\ N^{1 / p} & (1<p \leqslant 2,2 \leqslant q<\infty) \\ N^{1 / q} & (2 \leqslant p<\infty, 1<q \leqslant 2) \\ N^{1 / 2} & (2 \leqslant p<\infty, 2 \leqslant q<\infty)\end{cases}
$$

and

$$
\|b\|_{\infty, p, q} \leqslant K \begin{cases}N^{1 / p+1 / q-1 / 2} & (1<p \leqslant 2,1<q \leqslant 2), \\ N^{\max (1 / p, 1 / q)} & \text { elsewhere. }\end{cases}
$$

Since we have $\|a b\|_{2, p, q}=N^{1 / p+1 / q+1 / 2}$, the conclusion follows at once. We remark that the same method shows that $l^{2} \otimes l^{2}$ is not an $r$-summing algebra for any $1 \leqslant r<\infty$.

The Schur multiplication on $l^{p} \otimes_{\varepsilon} l^{q} \otimes_{\varepsilon} l^{r}(1 \leqslant p, q, r \leqslant \infty)$ is defined by $(a(k, \dot{m}, n)) \cdot(b(k, m, n))=(a(k, m, n) b(k, m, n)) \quad \forall a, b \in l^{p} \otimes_{\varepsilon} l^{q} \otimes_{\varepsilon} l^{r}$.

An important step in Varopoulos' proof of the existence of a commutative operator algebra which is not a $Q$-algebra was his theorem that $l^{2} \otimes_{8} l^{2} \otimes_{8} l^{2}$ is not a normed algebra. We propose to give a much simplified proof of this interesting fact in the course of an investigation of the general tensor algebras $l^{p} \otimes_{\varepsilon} l^{q} \otimes_{\varepsilon} l^{r}$.

The next proposition shows the interest of deciding when $l^{p} \ddot{\otimes} l^{\alpha}$ is an $r$-summing algebra for general $r$.

PropostTION 2.9. $l^{p} \otimes_{\varepsilon} q^{q} \otimes_{\varepsilon} l^{r}$ is a normed algebra if and only if $l^{p} \otimes l^{\alpha}$ is an $(r, r, r)$-summing algebra.

COROLLARY. If $l^{p} \otimes l^{q}$ is an r-summing algebra, then $l^{p} \otimes_{\varepsilon} l^{\alpha} \otimes_{\varepsilon} l^{r}$ is. a normed algebra.

Proof of Proposition 2.9. $l^{p} \ddot{\otimes} z^{\alpha}$ is an $(r, r, r)$-summing algebra if and only if there is some positive $K$ for which

$$
\sup _{\varphi}\left(\sum_{n}\left|\left\langle\tilde{\varphi}\left(a_{n}\right), b_{n}\right\rangle\right|^{r}\right)^{1 / r} \leqslant K \sup _{\psi}\left(\sum_{n}\left|\left\langle a_{n}, \psi\right\rangle\right|^{1 / r} \sup _{\theta}\left(\sum_{n}\left|\left\langle b_{n}, \theta\right\rangle\right|^{r}\right)^{1 / r}\right.
$$

for all finite sequences $a_{1}, \ldots, a_{N}$ and $b_{1}, \ldots, b_{N}$ in $l^{p} \otimes_{\varepsilon} l^{q}$. The suprema are taken over all $\varphi, \psi, \theta \in$ ball $\left(\left(l^{p} \otimes_{\varepsilon} l^{l}\right)^{\prime}\right)$.

Hoxistence of some positive $K$ for which

$$
\begin{aligned}
& \quad \sup \left\{\left(\sum_{n}\left|\sum_{k, m} a_{n}(k, m) b_{n}(k, m) x(k) y(m)\right|^{r}\right)^{1 / r}: x \in b^{p^{\prime}}, y \in b^{q^{\prime}}\right\} \\
& \leqslant K \cdot \sup _{x, y}\left[\sum_{n}\left|\sum_{k, m} a_{n}(k, m) x(k) y(m)\right|^{r}\right]^{1 / r} \sup _{x, y}\left[\sum_{n}\left|\sum_{k, m} b_{n}(k, m) x(k) y(m)\right|^{r}\right]^{1 / r}
\end{aligned}
$$


for all finite rank tensors $a=\left(a_{n}(k, m): 1 \leqslant k, m, n \leqslant N\right)$ and $b=\left(b_{n}(k, m)\right.$ : $1 \leqslant k, m, n \leqslant N)$. This, in turn, is equivalent to

$$
\|a b\|_{p, q, r} \leqslant K\|a\|_{p, q, r}\|b\|_{p, q, r}
$$

for all finite rank tensors $a$ and $b$.

If one of the indices $p, q$ or $r$ is 1 or $\infty$, it is clear that $l^{p} \otimes_{8} l^{a} \otimes_{B} l^{r}$ is a normed algebra. Apart from these trivial cases, our positive results may be summarised by the next two propositions.

Propostmion 2.10. If $1 / p+1 / q \geqslant 3 / 2$, then $l^{p} \otimes_{8} l^{q} \otimes_{8} l^{r}$ is a normed algebra for all $1 \leqslant r \leqslant \infty$.

By symmetry, the indices $p, q$ and $r$ may be freely interchanged in Proposition 2.10.

Proposition 2.11. If $1 \leqslant p, q, r \leqslant 2$ and $1 / p+1 / q+1 / r \geqslant 2$, then $l^{p} \otimes_{8} l^{q} \otimes_{8} l^{r}$ is a normed algebra.

Proof of Proposition 2.10. We suppose that $\|a\|_{p, q, r} \leqslant 1$ and $\|b\|_{p, q, r} \leqslant 1$. Since $1 / p+1 / q \geqslant 3 / 2$, the Hardy-Littlewood inequalities give

$$
\sup _{n}\left(\sum_{k, m}|a(k, m, n)|^{2}\right)^{1 / 2} \leqslant K
$$

On using Khintchin's inequality, the hypothesis on $b$ yields that

(12) $\sup \left\{\left(\sum_{n}\left(\sum_{k, m}|b(k, m, n) x(k) y(m)|^{2}\right)^{r / 2}\right)^{1 / r}: x \in b^{p^{\prime}}, y \in b^{q^{\prime}}\right\} \leqslant K$.

It follows from (11) and (12) that

$$
\begin{aligned}
& \|a b\|_{p, q, r}=\sup _{x, y}\left(\sum_{n}\left|\sum_{k, m} a(k, m, n) b(k, m, n) x(k) y(m)\right|^{r}\right)^{1 / r} \\
& \quad \leqslant \sup _{x, y}\left[\sum_{n}\left(\sum_{k, m}|a(k, m, n)|^{2}\right)^{r / 2} \cdot\left(\sum_{k, m}|b(k, m, n) x(k) y(m)|^{2}\right)^{r / 2}\right]^{1 / r} \leqslant K .
\end{aligned}
$$

We have to work a little harder to give the

Proof of Proposition 2.11. Suppose that $\|a\|_{p, q, r} \leqslant 1$ and $\|b\|_{p, a, r}$ $\leqslant 1$. Using Khintchin's inequality, we have

$$
\sup \left\{\left(\sum_{k}\left(\sum_{m, n}|a(k, m, n) y(m) z(n)|^{2}\right)^{p / 2}\right)^{1 / p}: y \in b^{q^{\prime}}, z \in b^{r^{\prime}}\right\} \leqslant K
$$

and

$$
\sup \left\{\left(\sum_{m}\left(\sum_{k, n}|a(k, m, n) x(k) z(n)|^{2}\right)^{\alpha / 2}\right)^{1 / q}: x \in b^{p^{\prime}}, z \in b^{r^{\prime}}\right\} \leqslant K .
$$

Fix $z \in b^{r^{\prime}}$, and write $A(k, m)=\left(\sum_{n}|a(k, m, n) z(n)|^{2}\right)^{1 / 2}$. Then

$$
\sup \left\{\left(\sum_{m}\left(\sum_{k}|A(k, m) x(k)|^{2}\right)^{q / 2}\right)^{1 / \alpha}: x \in b^{p^{\prime}}\right\} \leqslant K
$$

and

$$
\sup \left\{\left(\sum_{k_{k}}\left(\sum_{m}|A(k, m) y(m)|^{2}\right)^{p / 2}\right)^{1 / p}: y \in b^{q^{\prime}}\right\} \leqslant K .
$$

Setting $1 / u_{3}=1 / p+1 / q-1$, the arguments of Hardy and Littlewood may be followed verbatim to give

$$
\left[\sum_{k}\left(\sum_{m} A(k, m)^{2}\right)^{u_{3} / 2}\right]^{1 / u_{3}} \leqslant K \quad \text { and } \quad\left[\sum_{m}\left(\sum_{k} \mathcal{A}(k, m)^{2}\right)^{u_{3} / 2}\right]^{1 / u_{3}} \leqslant K,
$$

or, in other words,

$$
\left[\sum_{k}\left(\sum_{m, n}|a(k, m, n) z(n)|^{2}\right)^{u_{3} / 2}\right]^{1 / u_{3}} \leqslant K
$$

and

$$
\left[\sum_{m}\left(\sum_{k, n}|a(k, m, n) z(n)|^{2}\right)^{u_{3} / 2}\right]^{1 / u_{3}} \leqslant K .
$$

A symmetric argument proves that, if $y \in b^{q^{\prime}}$, then

$$
\left[\sum_{k}\left(\sum_{m, n}|a(k, m, n) y(m)|^{2}\right)^{u_{2} / 2}\right]^{1 / u_{2}} \leqslant K
$$

and

$$
\left[\sum_{n}\left(\sum_{k, m}|a(k, m, n) y(m)|^{2}\right)^{u_{2} / 2}\right]^{1 / u_{2}} \leqslant K
$$

where $1 / u_{2}=1 / p+1 / r-1$. Consequently,

$$
\begin{aligned}
& \|a b\|_{p, q, r}=\sup \left\{\left(\sum_{k}\left|\sum_{m, n} a(k, m, n) b(k, m, n) y(m) z(n)\right|^{p}\right)^{1 / p}: y \in b^{q^{\prime}}, z \in b^{r^{\prime}}\right\} \\
& \leqslant \sup _{y, z}\left[\sum_{k}\left(\sum_{m, n}|a(k, m, n) y(m)|^{2}\right)^{p / 2} \cdot\left(\sum_{m, n}|b(k, m, n) z(n)|^{2}\right)^{p / 2}\right]^{1 / p} \\
& \leqslant \sup _{y, z}\left[\sum_{k}\left(\sum_{m, n}|a(k, m, n) y(m)|^{2}\right)^{u_{2} / 2}\right]^{1 / u_{2}}\left[\sum_{k}\left(\sum_{m, n}|b(k, m, n) z(n)|^{2}\right)^{u_{3} / 2}\right]^{1 / u_{3}} \\
& \leqslant K, \quad \text { provided that } p / u_{2}+p / u_{3} \geqslant 1 .
\end{aligned}
$$

This last condition means that $1 / p+1 / q+1 / r \geqslant 2$.

Our final result in this section is

THEOREM 2.12. Let $1 \leqslant p, q, r \leqslant 2$. Then $l^{p} \otimes_{8} l^{q} \otimes_{8} l^{r}$ is a normed algebra if and only if $1 / p+1 / q+1 / r \geqslant 2$. 
Proof. After Proposition 2.11, we need only show that, if $1 / p+1 / q+$ $+1 / r<2$, then $l^{p} \otimes_{s} l^{\alpha} \otimes_{s} l^{r}$ is not a normed algebra. However, this follows immediately from Theorem 1.1. For there is a tensor $t=(t(k, m, n)$ : $1 \leqslant k, m, n \leqslant N)$ such that

$$
\|t\|_{p, q, r} \leqslant K N^{1 / p+1 / q+1 / r-1}
$$

If $l^{p} \otimes_{e} l^{\alpha} \otimes_{\varepsilon} l^{r}$ is to be a normed algebra, we must have

$$
N^{1 / p+1 / q+1 / r}=\left\|t^{2}\right\|_{p, q, r} \leqslant K\|t\|_{p, q, r}^{2} \leqslant K N^{2 / p+2 / q+2 / r-2}
$$

for some $K$ independent of $N$. This can only be true if $1 / p+1 / q+1 / r \geqslant 2$.

The same method only yields incomplete results for other values of $p, q$ and $r$. The diagrams below summarise our knowledge.
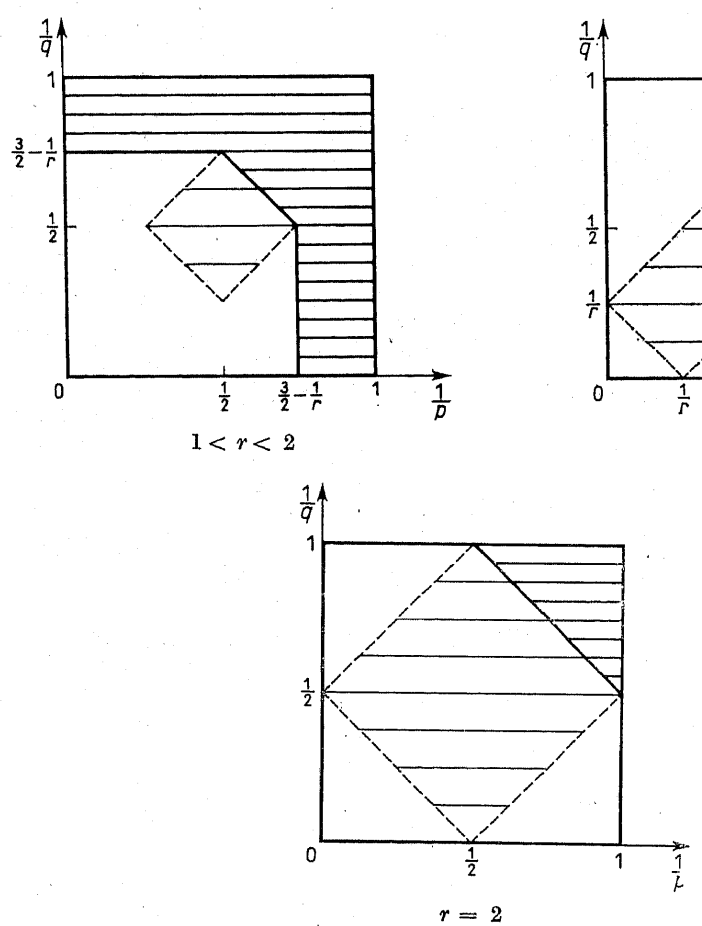

$l^{p} \otimes_{\varepsilon} l^{q} \otimes_{\varepsilon} l^{r}$ is a normed algebra in the heavily shaded regions; it is not a normed algebra in the lightly shaded regions; and the white regions are areas of uncertainty.

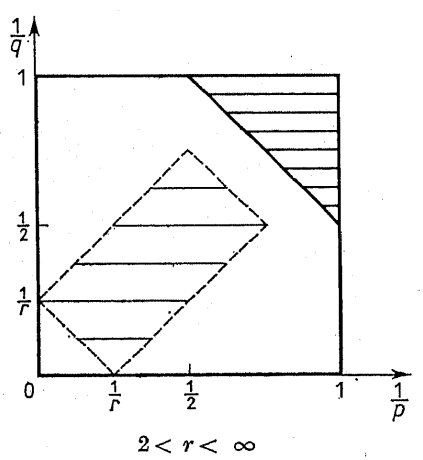
and

It is perhaps interesting to note that the multiplication on $l^{2} \otimes_{\varepsilon} l^{2} \otimes_{\varepsilon}{ }^{2}$ is, in a certain sense, badly non-continuous. Indeed, if we consider the product of $(a(k, m, n): 1 \leqslant k, m, n \leqslant N)$ and $(b(k, m, n): 1 \leqslant k, m, n$ $\leqslant N)$, we have

and this power of $N$ is best possible.

$$
\|a b\|_{2,2,2} \leqslant K N^{1 / 2}\|a\|_{2,2,2}\|b\|_{2,2,2},
$$

3. Absolutely summing and nuclear norms of random matrices. Let $t=(t(m, n): 1 \leqslant m, n<\infty)$ be an infinite matrix, and write $t_{N}$ for the $N \times N$ submatrix $(t(m, n): 1 \leqslant m, n \leqslant N)$. We shall interpret $t_{N}$ as an operator $l^{p}(N) \rightarrow l^{q}(N)(1 \leqslant p, q \leqslant \infty)$, and for $1 \leqslant r \leqslant \infty$ we shall denote its $r$-summing norm by $\pi_{r}\left(t_{N} ; p, q\right)$, its $r$-nuclear norm by $\nu_{r}\left(t_{N} ; p, q\right)$ and its $r$-factorisable norm by $\gamma_{r}\left(t_{N} ; p, q\right)$. See [17] and [19] for any necessary definitions.

Let $\varphi$ denote any one of the norms $\pi_{r}, v_{r}$ or $\gamma_{r}$. Then the asymptotic behaviour of $\varphi\left(t_{N} ; p, q\right)$ as $N$ tends to infinity may conveniently be described by the following two quantities.

(a) The upper limit order $\Lambda_{t}(\varphi ; p, q)$ of the matrix $t$ is defined to be

$$
\inf \left\{\mu: \varphi\left(t_{N} ; p, q\right) \leqslant K N^{\mu}\right\}
$$

(b) its lower limit order $\lambda_{t}(\varphi ; p, q)$ is defined to be

$$
\sup \left\{\mu: \varphi\left(t_{N} ; p, q\right) \geqslant k N^{\mu}\right\} \text {. }
$$

In these expressions, it is understood that the inequalities are to hold for all positive integers $N$, and that $k$ and $K$ should be independent of $N$.

Clearly, we always have $\lambda_{t}(\varphi ; p, q) \leqslant \Lambda_{t}(\varphi ; p, q)$. It is interesting to note that in the special case of the identity matrix $I=(\delta(m, n))$, we have the stronger result that $\lambda_{I}(\varphi ; p, q)=\Lambda_{I}(\varphi ; p, q)$ when $\varphi$ is $\pi_{r}$ or $v_{r}(1 \leqslant p, q, r \leqslant \infty)$. This is established implicitly in [9], [17] and [19], where the value of $\Lambda_{I}(\varphi ; p, q)$ is calculated. For further results, see [1].

The Littlewood matrices $A_{n}$ are defined inductively on spaces of dimension $2^{n}$ by

$$
A_{1}=\left(\begin{array}{rr}
1 & 1 \\
1 & -1
\end{array}\right) \quad \text { and } \quad A_{n+1}=\left(\begin{array}{rr}
A_{n} & A_{n} \\
A_{n} & -A_{n}
\end{array}\right) .
$$

Making the obvious modifications needed in the definitions of the upper and lower limit orders, Pietsch showed implicitly in [17] that $\lambda_{A}(\varphi ; p, q)$ $=\Lambda_{A}(\varphi ; p, q)$ when $\varphi$ is $\pi_{r}$ or $\nu_{r}(1 \leqslant r \leqslant \infty)$, and he evaluated $\Lambda_{A}\left(\pi_{r} ; p, q\right)$ and $\Lambda_{A}\left(v_{r^{\prime}} ; q, p\right)$, except in the region $2<p, q<r<\infty$. The referee has shown how to use the results of [9] and [19] to fill this gap. We give an analogue of his proof in Lemma 3.5 below. 
The object of this section is to observe that for "most" matrices $t$ with coefficients \pm 1 , the asymptotic behaviour of $\pi_{r}\left(t_{N} ; p, q\right)$ and $v_{r}\left(t_{N} ; p, q\right)$ follows that of the Littlewood matrices.

Let $t=(t(m, n): 1 \leqslant m, n \leqslant \infty)$ be a family of independent random variables such that

$$
\operatorname{prob}\{t(m, n)=1\}=\operatorname{prob}\{t(m, n)=-1\}=\frac{1}{2} .
$$

Write $\varphi$ for any one of the norms $\pi_{r}$ and $v_{r}(1 \leqslant r \leqslant \infty)$. Then we modify definitions (a) and (b) above:

(a)' The upper limit order $\Lambda_{t}(p ; p, q)$ is the infimum of those indices $\mu$ with the property that, for all $0<\delta<1$, there exists a positive constant $K$, independent of $N$, such that

$$
\operatorname{prob}\left\{\varphi\left(t_{N} ; p, q\right) \leqslant K N^{\mu}\right\} \geqslant \delta .
$$

(b) ${ }^{\prime}$ The lower limit order $\lambda_{t}(\varphi ; p, q)$ is the supremum of those indices $\mu$ with the property that, for all $0<\delta<1$, there exists a positive constant $k$, independent of $N$, such that

$$
\operatorname{prob}\left\{\varphi\left(t_{N} ; p, q\right) \geqslant k N^{\mu}\right\} \geqslant \delta .
$$

In each of the following propositions, $t$ denotes the random matrix defined above.

Proposition 3.1. If $1 \leqslant p, q, r \leqslant \infty$, then

$$
\lambda_{t}\left(\pi_{r} ; p, q\right)=\Lambda_{t}\left(\pi_{r} ; p, q\right) \quad \text { and } \quad \lambda_{t}\left(v_{r} ; p, q\right)=\Lambda_{t}\left(v_{r} ; p, q\right) \text {. }
$$

Moreover, $\Lambda_{t}\left(\pi_{r} ; p, q\right)=2-\Lambda_{t}\left(v_{r} ; q, p\right)$.

The values are given in the next three propositions.

Propostrion 3.2. If $1 \leqslant r \leqslant 2$, then $\Lambda_{t}\left(\pi_{r} ; p, q\right)$ is

(i) $1 / p^{\prime}+1 / 2$ for $1 \leqslant p \leqslant 2,2 \leqslant q \leqslant \infty$;

(ii) $1 / p^{\prime}+1 / q$ for $1 \leqslant p \leqslant r^{\prime}, 1 \leqslant q \leqslant 2$;

(iii) $1 / p^{\prime}+1 / q$ for $2 \leqslant q \leqslant p \leqslant r^{\prime}$

(iv) 1 for $2 \leqslant p \leqslant q \leqslant r^{\prime}$;

(v) 1 for $2 \leqslant p \leqslant \infty, r^{\prime} \leqslant q \leqslant \infty$; and

(vi) $1 / q+1 / r$ for $r^{\prime} \leqslant p \leqslant \infty, 1 \leqslant q \leqslant r^{\prime}$.

Proposimion 3.3. If $2<r<\infty$, then $\Lambda_{t}\left(\pi_{r} ; p, q\right)$ is

(i) $1 / p^{\prime}+1 / q$ for $1 \leqslant p \leqslant 2,1 \leqslant q \leqslant r$;

(ii) $1 / p^{\prime}+1 / r$ for $1 \leqslant p \leqslant r, r \leqslant q \leqslant \infty$;

(iii) $\left[\left(1 / p^{\prime}+1 / r\right)(1 / 2-1 / q)+(1 / q-1 / r)\right](1 / 2-1 / r)^{-1}$ for $2 \leqslant p, q \leqslant r$;

(iv) $1 / 2+1 / q$ for $2 \leqslant p \leqslant \infty, 1 \leqslant q \leqslant 2$; and

(v) 1 for $r \leqslant p \leqslant \infty, 2 \leqslant q \leqslant \infty$.
PROPOSITION 3.4. $\Lambda_{t}\left(\pi_{\infty} ; p, q\right)$ is

(i) $1 / p^{\prime}+1 / q-1 / 2$ for $2 \leqslant p \leqslant \infty, 1 \leqslant q \leqslant 2$;

(ii) $1 / q$ for $1 \leqslant p \leqslant 2,1 \leqslant q \leqslant p^{\prime}$; and

(iii) $1 / p^{\prime}$ for $q^{\prime} \leqslant p \leqslant \infty, 2 \leqslant q \leqslant \infty$.

Notice that Proposition 3.4 is nothing more than a weakened version of Theorems 1.1 and 1.2 with $J=2$. Greater precision may also be brought to Propositions 3.2 and 3.3 , but at the cost of sometimes introducing logarithmic factors. (See [1].)

We may express Propositions 3.2, 3.3 and 3.4 diagrammatically. In each diagram, we plot $1 / p$ horizontally and $1 / q$ vertically. In the diagrams on the left, we indicate the level lines of $\Lambda_{t}\left(\pi_{r} ; p, q\right)$, and in those on the right, the value of $\Lambda_{t}\left(\pi_{r} ; p, q\right)$.
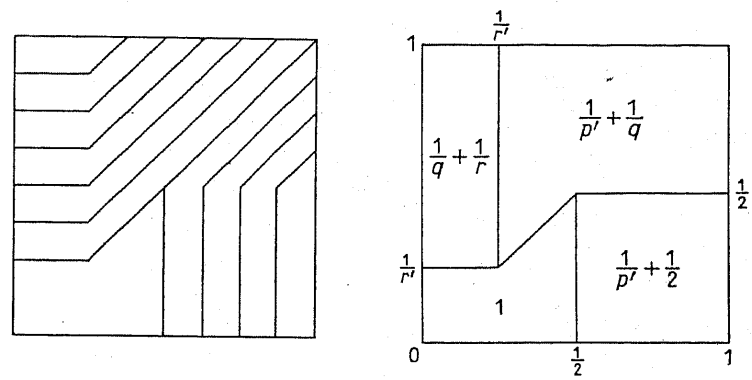

$1 \leqslant r \leqslant 2$
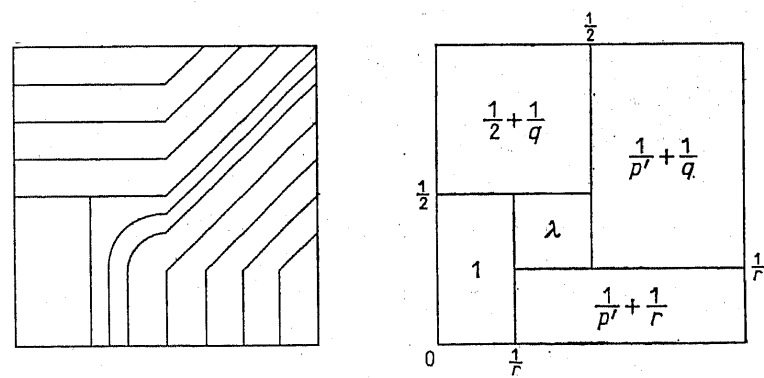

$\lambda=\frac{\left(\frac{1}{p^{\prime}}+\frac{1}{r}\right)\left(\frac{1}{2}-\frac{1}{q}\right)+\left(\frac{1}{q}-\frac{1}{r}\right)}{\left(\frac{1}{2}-\frac{1}{r}\right)}$ 

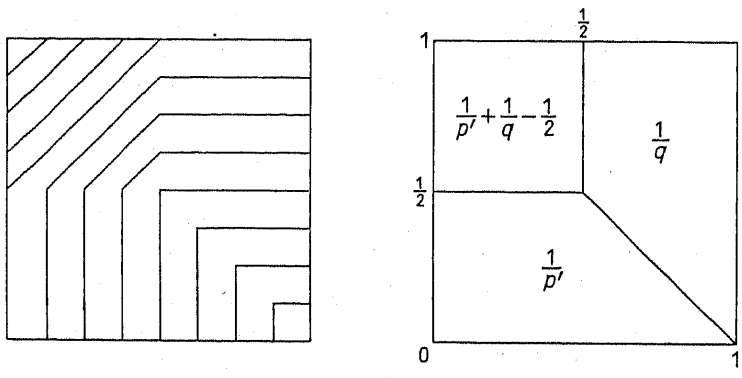

$r=\infty$

We omit most of the proofs of Propositions 3.1-3.4, as they are merely straightforward modifications of the arguments in [17]. In [17], Pietsch used the ideal property of $r$-summing and $r$-nuclear operators to exploit his knowledge of the behaviour of the identity operator. He was able to use the fact that $A_{n}$ is a multiple of a unitary matrix for his estimation of the norms of $A_{n}$, and the relation $A_{n}^{2}=2^{n} I_{2 n}$ showed his results to be precise. Our approach is to substitute Theorem 1.1 for the special properties of $A_{n}$. We need also to observe that

$$
N^{2}=\sum_{m, n} t_{N}(m, n) t_{N}(m, n)=\operatorname{trace}\left(t_{N}^{\prime} t_{N}\right) \leqslant v_{r^{\prime}}\left(t_{N}^{\prime} ; q, p\right) \pi_{r}\left(t_{N} ; p, q\right),
$$

and to note that prob $\{X \leqslant A\} \geqslant \eta$ and prob $\{X \leqslant B\} \geqslant \eta$ together imply that prob $\{X \leqslant A$ and $Y \leqslant B\} \geqslant 2 \eta-1$. Apart from this, the only novelty is the following lemma, for which we are endebted to the referee.

Lemara 3.5. Let $2<p, q<r<\infty$. Then and

(i) $\Lambda_{t}\left(\pi_{r} ; p, q\right)=\left[\left(1 / p^{\prime}+1 / r\right)(1 / 2-1 / q)+(1 / q-1 / r)\right](1 / 2-1 / r)^{-1}$ $+1 / p$.

(ii) $\Lambda_{t}\left(v_{r^{\prime}} ; q, p\right)=\left[1 / r^{\prime} \cdot(1 / 2-1 / q)+1 / p^{\prime} \cdot(1 / q-1 / r)\right](1 / 2-1 / r)^{-1}+$

Proof. We use the fact ([9], [19]) that, when $2<p, q<r<\infty$, (*)

$$
\Lambda_{I}\left(\gamma_{r} ; p, q\right)=(1 / 2-1 / p)(1 / q-1 / r)(1 / 2-1 / r)^{-1}
$$

(and $\left.\Lambda_{I}\left(\pi_{r} ; p^{\prime}, q\right)=1 / r+(1 / p-1 / r)(1 / q-1 / r)(1 / 2-1 / r)^{-1}\right)$. It will be enough to show that $\Lambda_{t}\left(\pi_{r} ; p, q\right)$ and $\Lambda_{t}\left(\nu_{r^{\prime}} ; q, p\right)$ are bounded above by the desired expressions.

(i) We know that $\Lambda_{t}\left(\pi_{r} ; p, 2\right)=1$ and that $\Lambda_{t}\left(\pi_{r} ; p, r\right)=1 / p^{\prime}+1 / r$. The bound for $\Lambda_{t}\left(\pi_{r} ; p, q\right)(2<q<r)$ now follows from an interpolation theorem of Carl [4]. (ii) We have

$$
\Lambda_{t}\left(v_{r^{\prime}} ; q, p\right) \leqslant \Lambda_{I}\left(v_{r^{\prime}} ; q, p^{\prime}\right)+\Lambda_{t}\left(\pi_{\infty} ; p^{\prime}, p\right)=\Lambda_{I}\left(v_{r^{\prime}} ; q, p^{\prime}\right)+1 / p .
$$
However,

$$
\begin{aligned}
\nu_{r^{\prime}}\left(I_{N} ; q, p^{\prime}\right) & =v_{r^{\prime}}\left(I_{N} ; q^{q}(N) \rightarrow l^{q^{\prime}}(N) \rightarrow l^{p^{\prime}}(N)\right) \\
& \leqslant \pi_{r^{\prime}}\left(I_{N}^{\prime} ; q, q^{\prime}\right) \gamma_{r^{\prime}}\left(I_{N} ; q^{\prime}, p^{\prime}\right) \quad(\text { by }[17], \text { Lemma } 4, \text { p. 298) } \\
& =\pi_{r^{\prime}}\left(I_{N} ; q, q^{\prime}\right) \gamma_{r}\left(I_{N} ; p, q\right) .
\end{aligned}
$$

The required inequality now follows from $(*)$, after a little calculation.

Finally, we should like to thank the referee for his helpful comments and for bringing several related papers to our attention.

\section{References}

[1] G. Baumbach and W. Linde, Asymptotic behaviour of p-absolutely summing norms of identity operators, Math. Nachr. 78 (1977), pp. 193-196.

[2] G. Bennett, V. Goodman and C. Newman, Norms of random matrices, Pacific J. Math. 59 (1975), pp. 359-365.

[3] R. C. Blei, A uniformity property for $A(2)$ sets and Grothendieck's inequality, Symposia Math., Vol. XXII (1977).

[4] B. Carl, A remark on p-integral and p-absolutely summing operators from $l_{u}$ into $l_{v}$, Studia Math. 57 (1976), pp. 257-262.

[5] B. Carl, B. Maurey, und J. P uhl, Grenzordnungen von absolut $(r, p)$-summierenden Operatoren, to appear.

[6] Ph. Charpentier, Sur les quotients d'algèbres uniformes, C. R. Acad. Sci. Paris 278 (1974), pp. 929-932.

[7] A. M. Davie, Quotient algebras of uniform algebras, J. London Math. Soc. 7 (1973), pp. 31-40.

[8] D. J. H. Garling, Diagonal mappings between sequence spaces, Studia Math. 51 (1974), pp. 129-138.

[9] E. D. Gluskin, A. Pietsch, and J. Puhl, A generalization of Khintchine's inequality and its application in the theory of operator ideals, ibid. 67 (1980), pp. 149-155.

[10] A. Grothendieck, Produits tensoriels topologiques et espaces nucléaires, Mem. Amer. Math. Soc. 16 (1955).

[11] G. H. Hardy, and J. E. Littlewood, Bilinear forms bounded in space $[p, q]$, Quart. J. Math. 5 (1934), pp. 241-254.

[12] J.-P. Kahane, Some random series of functions, Heath, 1968.

[13] J.-Th. Lapresté, Opérateurs sommants et factorisations à travers les espaces $L_{p}$, Studia Math. 57 (1976), pp. 47-83.

[14] J. Lindenstrauss, and A. Pełczyński, Absolutely summing operators in $\mathscr{L}_{p^{-}}$ spaces and their applications, Studia Math. 29 (1968), pp. 275-326.

[15] A. M. Mantero and A. M. Tonge, Banach algebras and von Neumann inequalities, Proc. London Math. Soc. 38 (1979), pp. 309-334.

[16] -, - Some remarks on the injective tensor product and Banach algebras, Boll. Un. Mat. Ital. (5) 16-A (1979), pp. 109-114.

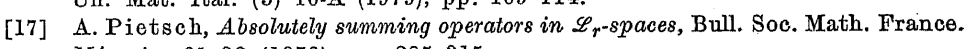
Mómoire 31-32 (1972), pp. 285-315. 
[18] - Absolut p-summierende Abbildungen in normierten Rüumen, Studia Math. 28 (1967), pp. 333-353.

[19] - Rosenthal's inequality and its application in the theory of operator ideals, (To appear in Proc. Leipzig Conference, 1977.)

[20] I. Schur, Bemerlcungen zur Theorie der beschränlten Bilinearformen mit unendlichen vielen Veränderlichen, J. für Math. 140 (1911), pp. 1-28.

[21] A. M. Tonge, Banach algebras and absolutely summing operators, Math. Proc. Cambridge Phil. Soc. 80 (1976), pp. 465-473.

[22] N. Th. Varopoulos, Some remarles on Q-algebras, Ann. Inst. Fourior (Grenoble) 22 (1972), pp. 1-11.

[23] - On an inequality of von Neumann and an application of the metric theory of tensor products to operator theory, J. Functional Analysis 16 (1974), pp. 83-100.

[24] - Sur le produit tensoriel des algebres normées, C. R. Acad. Sci. Paris 276 (1973), pp. 1193-1195.

[25] J. Wermer, Quotient algebras of uniform algebras, Symposium on Function Algebras and Rational Approximation, Michigan. 1969.

ISTITUTO DE MATEMATICA

UNIVERSTTA DI GENOVA, ITALY

and

DEPARTMENT OF PURE MATHEMATICS AND MATHIRMATICAL STATISTICS UNIVERSITY OF CAMBRIDGE, ENGLAND

Received April 22, 197\%

$(1300)$

\section{Fredholm Toeplitz operators on strongly pseudoconvex domains}

by

NICHOLAS P. JEWELL (Stanford, Cal.)

Abstract. Venugopalkrishna in [15] investigated conditions which ensure that a Toeplitz operator acting on a Hardy space on a strongly pseudoconvex domain $D \subseteq \boldsymbol{C}^{n}(n \geqslant 1)$ is Fredholm. In [11] McDonald proved that when $D=B^{n}$, the open unit ball in $\boldsymbol{C}^{n}$, then the Toeplitz operator $T_{\varphi}$, for $\varphi \in H^{\infty}+O$, is Fredholm if and only if $\varphi$ is bounded away from zero in a neighbourhood of $\partial B^{n}$. We extend this result to a general strongly pseudoconvex domain, $D$, with smooth boundary in $O^{n}$ with $n>2$, and give a similar result for Toeplitz operators acting on a Hardy space on $\partial D$. We also note that the property of a Toeplitz operator, $T_{\varphi}$, being Fredholm depends only on the local properties of the symbol $\varphi$ on $\partial D$.

1. Introduction. Let $D$ be a strongly pseudoconvex domain with smooth boundary in $C^{n}$, i.e., $D$ is a bounded domain in $C^{n}$ and there exists a real-valued function $\varrho$ such that

(1) $D=\{z: \varrho(z)<0\}$,

(2) $\operatorname{grad} \varrho \neq 0$ on $\partial D$,

(3) $\varrho$ is strictly plurisubharmonic in a neighbourhood of $\partial D$,

(4) $\varrho$ is of class $C^{\infty}$ in a neighbourhood of $\bar{D}$.

Denote by $L^{2}$ the space of functions $f: D \rightarrow C$ which are square integrable with respect to Lebesgue measure, $d \nabla$, in $C^{n}$. Write $L^{\infty}$ for the essentially bounded measurable functions on $D$. $H^{2}$ is the space of all functions $f \in L^{2}$ which are holomorphic in $D$, with norm $\|f\|_{2}=\left(\int_{D}|f(z)|^{2} d V(z)\right)^{1 / 2}$. $C$ is the space of all continuous functions on $\bar{D}$, and $A(\bar{D})$ is the space of all holomorphic functions in $D$ which extend continuously to $\bar{D}$. $H^{\infty}$ is the space of all bounded holomorphic functions on $D$.

Let $\sigma$ denote the surface area measure on $\partial D$. We write $L^{\infty}(\partial D)$ for $L^{\infty}(d \sigma), L^{2}(\partial D)$ for $L^{2}(d \sigma) . H^{2}(\partial D)$ denotes the closure in $L^{2}$ of the boundary values of holomorphic functions which extend smoothly to $\bar{D}$. Since the boundary of $D$ is smooth, this definition is equivalent to requiring that $\sup _{\varepsilon>0}\left[\int_{\partial D_{\varepsilon}}|f(z)|^{2} d \sigma_{\varepsilon}(z)\right]^{1 / 2}<\infty$ where $D_{\varepsilon}=\{z \in D: \varrho(z)<-\varepsilon\}, d \sigma_{\varepsilon}$ is surface area measure on $\partial D_{\varepsilon}$, and $f(z)$ is the Poisson integral extension of $f$ into $D$. The norm for $H^{2}(\partial D)$ is given by

$$
\|f\|_{2}=\left(\int_{\partial D}|f(z)|^{2} d \sigma(z)\right)^{1 / 2}
$$

